\title{
Angiotensin II Type 2 Receptor Signaling Attenuates Aortic Aneurysm in Mice Through ERK Antagonism
}

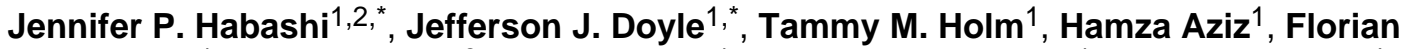 \\ Schoenhoff ${ }^{1}$, Djahida Bedja ${ }^{3}$, YiChun Chen ${ }^{1}$, Alexandra N. Modiri ${ }^{1}$, Daniel P. Judge ${ }^{4}$, and \\ Harry C. Dietz ${ }^{1,2,4, \dagger}$ \\ ${ }^{1}$ Howard Hughes Medical Institute and Institute of Genetic Medicine, Johns Hopkins University \\ School of Medicine, Baltimore, MD 21205, USA. \\ 2Division of Pediatric Cardiology, Department of Pediatrics, Johns Hopkins University School of \\ Medicine, Baltimore, MD 21205, USA. \\ ${ }^{3}$ Department of Molecular and Comparative Pathobiology, Johns Hopkins University School of \\ Medicine, Baltimore, MD 21205, USA. \\ ${ }^{4}$ Department of Medicine, Johns Hopkins University School of Medicine, Baltimore, MD21205, \\ USA.
}

\begin{abstract}
Angiotensin II (AngII) mediates progression of aortic aneurysm, but the relative contribution of its type 1 (AT1) and type 2 (AT2) receptors remains unknown. We show that loss of AT2 expression accelerates the aberrant growth and rupture of the aorta in a mouse model of Marfan syndrome (MFS). The selective AT1 receptor blocker (ARB) losartan abrogated aneurysm progression in the mice; full protection required intact AT2 signaling. The angiotensin-converting enzyme inhibitor (ACEi) enalapril, which limits signaling through both receptors, was less effective. Both drugs attenuated canonical transforming growth factor- $\beta$ (TGF $\beta$ ) signaling in the aorta, but losartan uniquely inhibited TGF $\beta$-mediated activation of extracellular signal-regulated kinase (ERK), by allowing continued signaling through AT2. These data highlight the protective nature of AT2 signaling and potentially inform the choice of therapies in MFS and related disorders.
\end{abstract}

Marfan syndrome (MFS) is an autosomal dominant connective tissue disorder that includes a predisposition for aortic root aneurysm and aortic rupture. MFS is caused by a deficiency of the microfibrillar constituent protein fibrillin-1 that is imposed by heterozygous mutations in $F B N 1$. In prior work, we demonstrated that transforming growth factor- $\beta$ (TGF $\beta$ ) signaling was elevated in affected tissues of mice heterozygous for a cysteine substitution in an epidermal growth factor-like domain of fibrillin-1 $\left(\mathrm{Fbnl} \mathrm{C}^{\mathrm{C} 1039 \mathrm{G} /+}\right)$, the most common class of mutation in people with MFS (1-4). Many disease manifestations-including aortic aneurysm (1), developmental emphysema (2), myxomatous degeneration of the atrioventricular valves (3), and skeletal muscle myopathy (4) — are attenuated by systemic

Copyright 2011 by the American Association for the Advancement of Science; all rights reserved.

${ }_{*}^{\dagger}$ To whom correspondence should be addressed. hdietz@jhmi.edu.

*These authors contributed equally to this work.

Supporting Online Material

www.sciencemag.org/cgi/content/full/332/6027/361/DC1

Materials and Methods

Figs. S1 to S12

References 
administration of a pan-specific polyclonal TGF $\beta$-neutralizing antibody (TGF $\beta N A b)$ in fibrillin-1-deficient mice. Similar protection was achieved by treating $\mathrm{Fbnl}^{\mathrm{Cl} 1039 \mathrm{G} /+}$ mice with the angiotensin II (AngII) type 1 (AT1) receptor blocker (ARB) losartan (1, 4). ARBs can attenuate TGF $\beta$ signaling in some tissues by lowering the expression of TGF $\beta$ ligands, receptors, and activators (5-7). In this mouse model of MFS, losartan's protection correlated with decreased phosphorylation and nuclear translocation of Smad2 (pSmad2), a direct effector of canonical TGF $\beta$ signaling, and decreased expression of prototypical Smaddependent TGF $\beta$-responsive gene products, such as connective tissue growth factor and collagens.

The contribution of AT2 to aortic aneurysm progression remains controversial. AT2 signaling can oppose AT1-mediated enhancement of TGF $\beta$ signaling in some cell types and tissues (Fig. 1A) (8, 9). It can also induce vascular smooth muscle cell (VSMC) apoptosis, theoretically contributing to aortic wall damage. Apoptosis was observed in cultured cells derived from end-stage aneurysms in people with MFS (10), but has not been found in earlyor intermediate-stage aortic wall lesions in MFS mice (1). Vascular expression of AT2 is largely limited to prenatal life, but it may continue to be relevant postnatally in the context of certain disease states, as evidenced by the acceleration of inflammatory aneurysms in AngII-infused mice treated with an AT2 antagonist (11). In contrast, $\beta$-aminopropionitrile monofumarate (BAPN)-induced aortic aneurysm and dissection in rats, which was associated with increased expression of AT2 and VSMC apoptosis, was ameliorated by limiting AngII production with angiotensin-converting enzyme inhibitor (ACEi) but not by selective AT1 receptor blockade (12). AT2 signaling has the capacity to attenuate both canonical (Smad-dependent) and noncanonical (mitogen-activated protein kinase or MAPK) TGF $\beta$ signaling cascades, most notably the extracellular signal-regulated kinase (ERK), in some tissues $(13,14)$. Thus, AT2 signaling can both augment and inhibit the pathogenesis of aneurysm in pre-clinical models, and the mechanistic explanation for the discordance is unclear. This has direct clinical relevance, as it leaves open to question the relative therapeutic merits of selective AT1 blockade with ARBs versus limiting signaling through both AT1 and AT2 with ACEi, despite small trials suggesting that either approach has potential in MFS (15-17).

To assess the role of the AT2 receptor in MFS, we bred mice with a disrupted Agtr2 allele (encoding AT2; AT2KO) $(18,19)$ with $F b n 1^{\mathrm{C} 1039 \mathrm{G} /+}$ mice, our established model of MFS (20). Agtr2 is encoded on the X chromosome in humans and mice, and the AT2KO allele associates with loss of mRNA and protein expression, as assessed by radioligand binding, in either homozygous females or hemizygous males. The AT2KO mice develop normally, with no evidence of cardiovascular pathology or early mortality (21).

We followed the progression of aortic root aneurysm by echocardiogram until the mice were killed at 12 months (Fig. 1B). There was a small difference in aortic root size between wildtype (WT) and AT2KO mice $(P<0.05)$ at 2 months, but this difference was absent at all future time points $(P=0.70)$. The aortic root diameter of AT2KO:Fbn ${ }^{\mathrm{C} 1039 \mathrm{G} /+}$ mice was significantly larger than that seen in $F b n l^{\mathrm{C} 1039 \mathrm{G} /+}$ mice at 2 months of age $(P<0.001)$, and this difference was maintained through to 12 months of life $(P<0.05)$. The postnatal aortic root growth over 10 months was not different between $F b n I^{\mathrm{C} 1039 \mathrm{G} /+}$ mice with or without AT2 expression $(P=0.80)$. This could reflect postnatal waning of AT2 receptor expression, attainment of an absolute threshold of aortic root growth rate in AT2KO:FbnI ${ }^{\mathrm{C} 1039 \mathrm{G} /+}$ mice, and/or the accelerated death observed in AT2KO: $F b n I^{\mathrm{C} 1039 \mathrm{G} /+}$ mice that effectively removed the most severely affected animals from later analyses. We found that $32 \%$ of AT2KO:Fbn $1^{\mathrm{C} 1039 \mathrm{G} /+}$ mice died before the scheduled killing, compared with $12 \%$ of Fbn $1^{\mathrm{C} 1039 \mathrm{G} /+}$ mice $(P<0.01)$ and $0 \%$ of AT2KO or WT mice (Fig. 1C). Growth of the more distal ascending aorta over 10 months was significantly greater in 
AT2KO:Fbnl ${ }^{\mathrm{C} 1039 \mathrm{G} /+}$ mice compared with $F b n l^{\mathrm{C} 1039 \mathrm{G} /+}$ littermates $(P<0.05)$, whereas there was no significant difference between WT, AT2KO, and $F b n I^{\mathrm{C} 1039 \mathrm{G} /+}$ mice (Fig. 1D).

Histological and morphometric analyses of the aortic media were performed at 12 months. AT2KO:Fbnl ${ }^{\mathrm{C} 1039 \mathrm{G} /+}$ mice showed medial thickening, reduced elastin content, and increased elastic fiber fragmentation (Fig. 2A and figs. S1 to S3) compared with $\mathrm{Fbnl}^{\mathrm{C} 1039 \mathrm{G} /+}$ or AT2KO mice $(P<0.01$ for all comparisons). These parameters were not significantly different in AT2KO and WT mice $(P=0.07, P=0.68$, and $P=1.0$, respectively). Therefore, the histological changes in the aorta paralleled the echocardiography findings, which supported the conclusion that AT2 receptor elimination exacerbates aortic disease in MFS mice.

The potential for exacerbation of the MFS phenotype outside of the cardiovascular system was also assessed. At 12 months, excised lungs were inflated with agar, sectioned, and stained for histological and morphometric analyses (figs. S4 and S5). Increased distal airspace caliber, a marker of impaired distal alveolar septation and emphasematous lung disease, can be quantified by calculating a mean linear intercept (MLI). There was no significant difference in MLI between WT and AT2KO mice $(P=1.0)$. Compared with WT and AT2KO littermates, $F b n I^{\mathrm{C} 1039 \mathrm{G} /+}$ mice had a significant increase in MLI $(P<0.05)$, whereas AT2KO:FbnI $\mathrm{C1039 \textrm {G } / +}$ mice had a yet further increase in MLI $(P<0.05)$. This confirms that AT2 receptor elimination can exacerbate the MFS phenotype outside of the cardiovascular system.

We next performed a head-to-head comparison of ACEi versus ARBs. FbnI C1039G/+ mice and WT littermates were treated with hemodynamically equivalent doses (fig. S6) of either the ACEi enalapril (10 to $15 \mathrm{mg} / \mathrm{kg}$ of body weight per day) or the ARB losartan (40 to 60 $\mathrm{mg} / \mathrm{kg}$ per day) (22), beginning at 8 weeks of age, and were assessed with serial echocardiograms. Aortic root growth over the 7 months of treatment was significantly greater in placebo-treated $F b n l^{\mathrm{C} 1039 \mathrm{G} /+}$ mice compared with WT littermates $(P<0.01)$, whereas losartan led to a significant regression in growth in $F b n l^{\mathrm{C} 1039 \mathrm{G} /+}$ mice $(P<$ $0.0001)$, to rates that were significantly less than that seen in WT littermates $(P<0.0001)$ (1). It is noteworthy that losartan reduced aortic root growth in $F b n I^{\mathrm{C} 1039 \mathrm{G} /+}$ mice, but had no effect in WT littermates $(P=0.27)$. Enalapril treatment had significantly less effect than losartan in $F b n l^{\mathrm{C} 1039 \mathrm{G} /+}$ mice $(P<0.0001)$; in fact, it was only marginally better than placebo treatment $(P=0.05)$ (Fig. 2B). Enalapril was also no more beneficial than placebo in improving aortic architecture score in $F b n I^{\mathrm{C} 1039 \mathrm{G} /+}$ mice $(P=0.19)$, whereas losartan was significantly more beneficial than both placebo and enalapril treatment $(P<0.05$ for both) (fig. S7).

We next assessed whether AT2 signaling is needed to achieve losartan's full therapeutic benefit. AT2KO:FbnI ${ }^{\mathrm{C} 1039 \mathrm{G} /+}$ mice were treated with losartan from 8 weeks of age and followed by serial echocardiography until they were killed at 9 months of age (Fig. 2C). Although there was a trend for increased aortic root growth in AT2KO:FbnI ${ }^{\mathrm{C} 1039 \mathrm{G} /+}$ mice compared with $F b n l^{\mathrm{C} 1039 \mathrm{G} /+}$ littermates $(P=0.06)$, the decrease in aortic root growth seen in AT2KO:Fbnl ${ }^{\mathrm{C} 1039 \mathrm{G} /+}$ mice treated with losartan was only $40 \%$ of that seen in FbnI ${ }^{\mathrm{C} 1039 \mathrm{G} /+}$ animals that expressed AT2 $(P<0.001)$, despite there being no difference in blood pressure between the groups (Fig. $2 \mathrm{C}$ and fig. S6). The modest reduction in aortic root growth seen in losartan-treated AT2KO:FbnI ${ }^{\mathrm{C} 1039 \mathrm{G} /+}$ mice was comparable to that previously observed with propranolol, and it may be similarly attributable to a decline in blood pressure rather than a modulation of cytokine signaling (1).

Together, these experiments suggest that AT2 signaling protectively modifies MFS and that the therapeutic effect of ACEi likely relates to AT1 receptor blockade or antihypertensive 
effects. In addition, we have shown that selective AT1 antagonism with the ARB losartan is beneficial in $\mathrm{Fbnl}^{\mathrm{C} 1039 \mathrm{G} /+}$ mice and that AT2 signaling is needed to achieve the full potential of ARBs. Both the canonical (Smad-dependent) and non-canonical (MAPK, predominantly ERK1/2 but also JNK in some experimental contexts) TGF $\beta$ signaling cascades are activated in $\mathrm{Fbnl} \mathrm{C}^{\mathrm{C} 1039 \mathrm{G} /+}$ mice in a TGF $\beta$ - and AT1 receptor-dependent manner (23). To investigate the mechanism of protection by AT2 receptor signaling, we monitored the status of both canonical and noncanonical TGF $\beta$ signaling in $\mathrm{Fbnl}$ C1039G/+ mice lacking the AT2 receptor or in response to losartan or enalapril treatment. Western blot analysis showed that Smad2 activation was significantly greater in the aortic root and proximal ascending aorta of $F b n l^{\mathrm{C} 1039 \mathrm{G} /+}$ mice compared with WT controls $(P<0.01)$ but that there was no significant difference between AT2KO:Fbn ${ }^{\mathrm{C} 1039 \mathrm{G} /+}$ and $\mathrm{Fbn} \mathrm{I}^{\mathrm{C} 1039 \mathrm{G} /+}$ mice $(P=0.30)$. In contrast, ERK1/2 activation was significantly greater in $F b n l^{\mathrm{C} 1039 \mathrm{G} /+}$ mice compared with WT littermates $(P<0.01)$ and was further increased in AT2KO:Fbn $1^{\mathrm{C} 1039 \mathrm{G} /+}$ mice compared with $F b n 1^{\mathrm{C} 1039 \mathrm{G} /+}(P<0.01)$, AT2KO $(P<0.01)$, and WT littermates $(P<0.001)$ (Fig. 3A). The difference in ERK1/2 activation was specific to the aortic root and proximal ascending aorta, the areas most predisposed to aneurysm formation in MFS, as there was no significant difference in the descending thoracic aortas of the same animals (fig. S8). No significant differences in JNK1 or p38 activation were observed (fig. S9).

In our comparison of $\mathrm{ARBs}$ versus $\mathrm{ACEi}, \mathrm{Smad} 2$ activation was significantly greater in Fbn $1^{\mathrm{C} 1039 \mathrm{G} /+}$ mice compared with WT controls $(P<0.05)$, and losartan treatment significantly decreased Smad2 activation in $F b n 1^{\mathrm{C} 1039 \mathrm{G} /+}$ mice $(P<0.05)$ to levels indistinguishable from WT $(P=0.31)$ (Fig. 3B). Enalapril reduced Smad2 activation in $\mathrm{Fbnl} \mathrm{C}^{\mathrm{C} 1039 \mathrm{G} /+}$ mice significantly more than losartan $(P<0.01)$, a finding that did not parallel the therapeutic effects of these agents (Fig. 2B). ERK1/2 activation was significantly greater in $F b n 1^{\mathrm{C} 1039 \mathrm{G} /+}$ mice compared with WT controls $(P<0.01)$, and treatment with losartan reduced it to WT levels $(P=0.80)$. In contrast, enalapril treatment had significantly less effect on ERK1/2 activation than losartan $(P<0.001)$; in fact, it was no more effective than placebo $(P=0.50)$. JNK1 and p38 activation was similar in $F b n l^{\mathrm{C} 1039 \mathrm{G} /+}$ and WT mice; both losartan and enalapril caused a modest reduction in JNK1 activation $(P<0.01)$, but neither had any effect on p38 activation (fig. S10). Thus, the biochemical status of the noncanonical ERK1/2, but not the canonical Smad, TGF $\beta$ signaling cascade correlated with the therapeutic effects of these agents. In keeping with this finding, losartan had a reduced ability to lower ERK1/2 activation in $F b n l^{\mathrm{C} 1039 \mathrm{G} /+}$ mice lacking the AT2 receptor $(P<$ 0.01) (Fig. 3C). By contrast, there was no significant difference in Smad2, JNK1, or p38 activation in losartan-treated $\mathrm{Fbnl} \mathrm{C}^{\mathrm{C} 1039 \mathrm{G} /+}$ mice that did or did not express AT2 (fig. S11).

To assess for a contribution of other components of the renin-angiotensin-aldosterone system, we treated $\mathrm{Fbnl} \mathrm{C}^{\mathrm{C} 1039 \mathrm{G} /+}$ mice with the aldosterone receptor antagonist spironolactone (24). We found no significant inhibition of aortic root growth over 7 months time $(P=0.23)$ (fig. S12).

In conclusion, dual blockade of AT1 receptor-mediated ERK1/2 activation and AT2 receptor-mediated ERK1/2 inhibition, as occurs either with the use of ACEi in Fbn ${ }^{\mathrm{C} 1039 \mathrm{G} /+}$ mice or the use of losartan in AT2KO:Fbnl ${ }^{\mathrm{C} 1039 \mathrm{G} /+}$ mice, results in no net change in ERK1/2 activation status and adds a very modest therapeutic benefit. By contrast, losartan reduces ERK1/2 phosphorylation through a combination of both inhibiting AT1 receptor-mediated ERK activation and by shunting AngII signaling through the AT2 receptor. This indicates that, in the presence of AT1 receptor blockade, ongoing AT2 receptor signaling is required for the attenuation of ERK phosphorylation and that enalapril's lack of effect on ERK is attributable to the loss of AT2 receptor signaling potential with this agent (Fig. 3D). Given that the small reduction in aortic root growth in Fbn ${ }^{\mathrm{C} 1039 \mathrm{G} /+}$ mice 
achieved by enalapril in this study was comparable to that achieved previously by propanolol (1), this suggests that its small beneficial effect may well have been mediated by blood pressure reduction. Although the concordant effects of prior manipulations and therapies on canonical and noncanonical TGF $\beta$ signaling made it impossible to dissect their relative contributions, the differential effects of enalapril treatment suggest that TGF $\beta$ mediated ERK1/2 activation is the predominant driver of aneurysm progression in MFS. In light of this, analysis of ERK1/2 activation status may permit the optimization of dosing regimens for losartan or other ARBs in ongoing or future clinical trials in people with MFS. Furthermore, focused attention on the ERK1/2 signaling cascade may unveil new therapeutic targets in the treatment of aortic aneurysm disease.

\section{Supplementary Material}

Refer to Web version on PubMed Central for supplementary material.

\section{Acknowledgments}

Supported by the NIH (H.C.D., D.P.J.); Howard Hughes Medical Institute (H.C.D.); the National Marfan Foundation (H.C.D., J.P.H., J.J.D.); the Cellular and Molecular Medicine Training Program, Johns Hopkins School of Medicine (J.J.D.); and the Smilow Center for Marfan Syndrome Research (H.C.D.). We thank T. Inagami for the AT2KO mice. Johns Hopkins University and the authors (H.C.D., J.P.H., D.P.J.) have filed a patent relating to the use of TGF $\beta$ antagonists, including AT1 receptor blockers, for the treatment of Marfan syndrome.

\section{References and Notes}

1. Habashi JP, et al. Science. 2006; 312:117. [PubMed: 16601194]

2. Neptune ER, et al. Nat. Genet. 2003; 33:407. [PubMed: 12598898]

3. Ng CM, et al. J. Clin. Invest. 2004; 114:1586. [PubMed: 15546004]

4. Cohn RD, et al. Nat. Med. 2007; 13:204. [PubMed: 17237794]

5. Wolf G, Ziyadeh FN, Stahl RA. J. Mol. Med. 1999; 77:556. [PubMed: 10494801]

6. Fukuda N, et al. Am. J. Hypertens. 2000; 13:191. [PubMed: 10701820]

7. Naito T, et al. Am. J. Physiol. Renal Physiol. 2004; 286:F278. [PubMed: 14583433]

8. Jones ES, Black MJ, Widdop RE. J. Mol. Cell. Cardiol. 2004; 37:1023. [PubMed: 15522279]

9. Rodríguez-Vita J, et al. Circulation. 2005; 111:2509. [PubMed: 15883213]

10. Nagashima H, et al. Circulation. 2001; 104(suppl. 1):I282. [PubMed: 11568070]

11. Daugherty A, Manning MW, Cassis LA. Br. J. Pharmacol. 2001; 134:865. [PubMed: 11606327]

12. Nagashima H, et al. J. Vasc. Surg. 2002; 36:818. [PubMed: 12368744]

13. Ulmasov B, Xu Z, Tetri LH, Inagami T, Neuschwander-Tetri BA. Am. J. Physiol. Gastrointest. Liver Physiol. 2009; 296:G284. [PubMed: 19033539]

14. Akishita M, et al. J. Clin. Invest. 1999; 103:63. [PubMed: 9884335]

15. Brooke BS, et al. N. Engl. J. Med. 2008; 358:2787. [PubMed: 18579813]

16. Yetman AT, Bornemeier RA, McCrindle BW. Am. J. Cardiol. 2005; 95:1125. [PubMed: 15842990]

17. Ahimastos AA, et al. JAMA. 2007; 298:1539. [PubMed: 17911499]

18. Materials and methods are available as supporting material on Science Online.

19. Ichiki T, et al. Nature. 1995; 377:748. [PubMed: 7477267]

20. Judge DP, et al. J. Clin. Invest. 2004; 114:172. [PubMed: 15254584]

21. Siragy HM, Inagami T, Ichiki T, Carey RM. Proc. Natl. Acad. Sci. U.S.A. 1999; 96:6506. [PubMed: 10339618]

22. Patten RD, et al. Clin. Sci. 2003; 104:109. [PubMed: 12546633]

23. Holm T, et al. Science. 2011; 332:358. [PubMed: 21493862]

24. Sakurabayashi-Kitade S, et al. Atherosclerosis. 2009; 206:54. [PubMed: 19327775] 


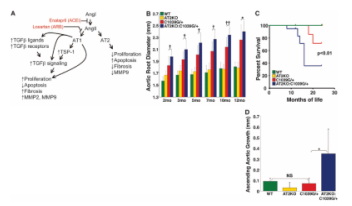

Fig. 1.

The role of AngII in the aorta. (A) AngII acts on the AT1 receptor causing increased cellular proliferation, fibrosis, and matrix metalloproteinase-2and/or -9 (MMP2/9) activity while decreasing apoptosis. Conversely, the AT2 receptor is thought to decrease proliferation, fibrosis, and MMP activity, while increasing apoptosis. ACEi's block the conversion of AngI to AngII, limiting signaling through both AT1 and AT2 receptors, whereas ARBs selectively block AT1. (B) Average absolute aortic root diameter $( \pm 2 \mathrm{SEM})$ measured serially by echo-cardiogram over the first year of life. Note that AT2KO:Fbn ${ }^{\mathrm{C} 1039 \mathrm{G} /+}$ mice have a significantly larger aortic root diameter than $\mathrm{Fbnl}^{\mathrm{C} 1039 \mathrm{G} /+}$ mice at each time point. (C) Kaplan-Meier survival curve demonstrating an increased rate of death in AT2KO:Fbn $1^{\mathrm{C} 1039 \mathrm{G} /+}$ as compared with $\mathrm{Fbnl} \mathrm{C}^{\mathrm{C} 1039 \mathrm{G} /+}$ mice. (D) Ascending aortic growth from 2 to 12 months of age. Note the increased rate of ascending aortic growth in AT2KO:Fbnl ${ }^{\mathrm{C} 1039 \mathrm{G} /+}$ mice. Final absolute ascending aortic diameter: WT $(1.41 \pm 0.07$ $\mathrm{mm}), \operatorname{AT} 2 \mathrm{KO}(1.40 \pm 0.07 \mathrm{~mm}), F b n l^{\mathrm{C} 1039 \mathrm{G} /+}(1.42 \pm 0.20 \mathrm{~mm}), \mathrm{AT} 2 \mathrm{KO}: F b n l^{\mathrm{C} 1039 \mathrm{G} /+}$ $(1.72 \pm 0.42 \mathrm{~mm})$. (B to D) WT $(n=5), \mathrm{AT} 2 \mathrm{KO}(n=10), F b n l^{\mathrm{C} 1039 \mathrm{G} /+}(n=17)$, AT2KO:Fbnl ${ }^{\mathrm{C} 1039 \mathrm{G} /+}(n=19) . * P<0.05 ; \dagger P<0.001$; $\dagger \dagger P<0.0001$; NS, not significant. 


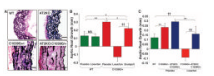

Fig. 2.

Therapeutic effects in the aorta. (A) WT $(n=5)$, AT2KO $(n=4), F b n 1^{\mathrm{C} 1039 \mathrm{G} /+}(n=7)$, and AT2KO:Fbnl ${ }^{\mathrm{C} 1039 \mathrm{G} /+}(n=7)$ mice. Verhoeff-Van Gieson (VVG) stain reveals diffuse fragmentation of elastic fibers and thickening of the media in $\mathrm{Fbnl} \mathrm{C}^{\mathrm{C} 1039 \mathrm{G} /+}$ mice; these findings are exaggerated in AT2KO:Fbn $\mathrm{C}^{\mathrm{C} 1039 \mathrm{G} /+}$ mice. (B) Average aortic root growth (T2SEM) over 7 months of treatment in placebo- $(n=13)$ orlosartan- $(n=7)$ treated WT mice and placebo- $(n=17)$, losartan- $(n=5)$, or enalapril- $(n=15)$ treated Fbn $1^{\mathrm{C} 1039 \mathrm{G} /+}$ mice, as measured by echocardiography. Note the regression in aortic size observed in losartan-treated $\mathrm{Fbnl} \mathrm{C}^{\mathrm{C} 1039 \mathrm{G} /+}$ mice and the marginal $(P=0.05)$ decrease in growth in the enalapril-treated cohort. Final absolute aortic root diameter: WT (1.74 $\pm 0.10 \mathrm{~mm})$, losartantreated WT $(1.77 \pm 0.15 \mathrm{~mm}), F b n l^{\mathrm{C} 1039 \mathrm{G} /+}(2.19 \pm 0.19 \mathrm{~mm})$, losartan-treated Fbn $1^{\mathrm{C} 1039 \mathrm{G} /+}(1.96 \pm 0.09 \mathrm{~mm})$, and enalapril-treated $F b n l^{\mathrm{C} 1039 \mathrm{G} /+}(2.18 \pm 0.18 \mathrm{~mm}) .(\mathbf{C})$ Average aortic root growth $( \pm 2 \mathrm{SEM})$ over 7 months of treatment in WT $(n=8)$, placebo- $(n$ $=22)$, and losartan- $(n=11)$ treated $F b n l^{\mathrm{C} 1039 \mathrm{G} /+}$ mice and placebo- $(n=19)$ and losartan$(n=6)$ treated AT2KO:FbnI ${ }^{\mathrm{C} 1039 \mathrm{G} /+}$ mice. Note the diminished effectiveness of losartan treatment in AT2KO:Fbnl ${ }^{\mathrm{C} 1039 \mathrm{G} /+}$ mice, as compared with losartan treatment in Fbn $1^{\mathrm{C} 1039 \mathrm{G} /+}$ mice. Final absolute aortic root diameter: WT $(1.77 \pm 0.10 \mathrm{~mm})$, Fbn $1^{\mathrm{C} 1039 \mathrm{G} /+}(2.13 \pm 0.16 \mathrm{~mm}), \mathrm{AT} 2 \mathrm{KO}: \mathrm{Fbnl}^{\mathrm{C} 1039 \mathrm{G} /+}(2.34 \pm 0.13 \mathrm{~mm})$, losartan-treated Fbn ${ }^{\mathrm{C} 1039 \mathrm{G} /+}(1.96 \pm 0.09 \mathrm{~mm})$, and losartan-treated AT2KO:Fbnl $\mathrm{C} 1039 \mathrm{G} /+(2.06 \pm 0.07$ $\mathrm{mm}) . * P<0.05 ; * * P<0.01 ; \dagger P<0.001 ; \dagger \dagger P<0.0001$; NS, not significant. 


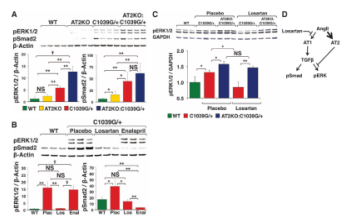

Fig. 3.

Mechanism of protection by AT2 signaling. (A) Western blot analysis of ERK1/2 and Smad2 activation in the aortic root and proximal ascending aorta of four mice of each genotype. Note that Smad2 activation is increased equally in AT2KO:FbnI ${ }^{\mathrm{C} 1039 \mathrm{G} /+}$ and Fbn $1^{\mathrm{C} 1039 \mathrm{G} /+}$ mice, compared with WT littermates. ERK1/2 activation is significantly increased in $\mathrm{Fbnl} \mathrm{C}^{\mathrm{C} 1039 \mathrm{G} /+}$ mice when compared with WT littermates and is further increased in AT2KO:FbnI ${ }^{\mathrm{C} 1039 \mathrm{G} /+}$ mice. (B) Western blot analysis of ERK1/2 and Smad2 activation in the aortic root and proximal ascending aortas of three each of WT and placebo-, losartan- or enalapril-treated $\mathrm{Fbnl} \mathrm{C}^{\mathrm{C} 1039 \mathrm{G} /+}$ mice. Note that Smad2 activation is decreased in both losartan- and enalapril-treated $\mathrm{Fbnl} \mathrm{C}^{\mathrm{C} 1039 \mathrm{G} /+}$ mice when compared with placebo-treated animals, with a more pronounced effect in enalapril-treated animals. In contrast, enalapril treatment failed to reduce ERK1/2 activation, whereas losartan reduced ERK1/2 activation to levels indistinguishable from WT littermates. (C) Western blot analysis of ERK1/2 activation in the aortic root and proximal ascending aorta of three WT, AT2KO, and placebo- or losartan-treated AT2KO:Fbn $I^{\mathrm{C} 1039 \mathrm{G} /+}$ mice. Note that losartan loses its ability to decrease ERK1/2 activation in AT2KO:Fbn $1^{\mathrm{C} 1039 \mathrm{G} /+}$ mice, demonstrating that the inhibition of ERK1/2 activation is mediated by the AT2 receptor. (D) Summary of the effects of AngII receptors on both canonical and noncanonical TGF $\beta$ signaling. AT1 receptor stimulation drives ERK1/2 activation, whereas AT2 receptor stimulation inhibits it. Losartan attenuates ERK1/2 activation by blocking the AT1 cascade while simultaneously shunting signaling through the AT2 receptor. ${ }^{*} P<0.05$; $* * P<0.01 ; \dagger P<0.001$; NS, not significant. 